\title{
Using Limited Feedback in Power Allocation Design for a Two-hop Relay OFDM System
}

\author{
Mahdi Hajiaghayi ${ }^{\dagger}$, Min Dong ${ }^{\ddagger}$ and Ben Liang ${ }^{\dagger}$ \\ $\dagger$ Dept. of Electrical and Computer Engineering, University of Toronto, CANADA \\ $\ddagger$ Faculty of Engineering and Applied Science, University of Ontario Institute of Technology, CANADA. \\ ${ }_{\dagger}$ Email: $\left\{\right.$ mahdih, liang\}@ comm.utoronto.ca, ${ }^{\ddagger}$ Email: min.dong@uoit.ca
}

\begin{abstract}
In this paper, we study power allocation (PA) in a single-relay OFDM system with limited feedback. We propose a PA scheme that uses a codebook of quantized PA vectors designed offline and known to the source, relay, and destination. The destination, which has full knowledge of channel side information (CSI), chooses one of the codebook vectors and conveys back to the source and relay. With the limited amount of available feedback, the design of an appropriate codebook is central to PA, which varies depending on the destination's strategy to choose the optimal PA vector. Assuming high received SNR on either link in the relay path, we first derive the optimal PA solutions as the function of channel realizations with two design criteria, maximizing capacity and minimizing error rate. It is found that when there is high received SNR in either the relay path or the direct path, the optimal solutions for both criteria reduce to simple forms. For maximizing capacity, the available power should be equally allocated to each OFDM subcarrier shared by the source and relay; while for minimum error rate, the available power should be allocated such that the received SNRs for all subcarriers at the destination are the same. The findings lead us to the sub-optimal solutions with great complexity reduction. We then present an adaptation of Lloyd's algorithm to construct a codebook to quantize the optimal PA vectors subject to the amount of feedback. Simulations show that a mild to negligible performance loss can be achieved with only a few bits of feedback at different SNR values.
\end{abstract}

\section{INTRODUCTION}

In wireless networking, both capacity and transmission range can be improved by relaying technique, where, for instance, a faraway or deeply shadowed user can be served with the help of relays that have a more favorable connection with the base station. Meanwhile, Orthogonal Frequency Division Multiplexing (OFDM) is a proven technology that utilizes broadband-frequency resources efficiently. It mitigates the problems of inter-symbol interference by dividing a broadband into a series of subcarriers and simultaneously transmitting parallel substreams through the subcarriers. In both technologies, appropriate power allocation (PA) is essential to optimal performance. In a system that combines both relaying and OFDM, PA refers to the allocation of the total transmission power among the source and relays and among the subcarriers. Clearly, depending on the system performance criteria, the type and number of relays, and the channel statistics, the optimal PA scheme would be different.

In [1] and [2], optimal PA for a narrow-band system between a source and a single Decode-and-Forward (DF)

This work is funded in part by LG Electronics. relay was investigated, where they share a limited budget of power. While these works considered a single-relay system, authors in [3] considered a system with multiple Amplifyand-Forward (AF) relays for an optimal PA solution to maximize the overall capacity. However, the increase of capacity obtained from properly assigning the power resources came at the price of obtaining the full knowledge of channel state information (CSI) at the relays and source. [4] addressed this questionable assumption by considering only the availability of the statistical property of CSI at the relays. However, the PA solution in [4] did not adapt to the channel variation in time.

For optimal system performance, the vector of allocated power among the nodes and the subcarriers (henceforth termed $P A$ vector) should vary in time according to the channel state. There are two approaches to achieve this in a system with limited feedback. One can either send a quantized version of CSI or send the quantized PA vectors through the feedback link. Based on the former method, the authors of [5] presented a limited-feedback PA scheme for a single relay system. This approach requires a large number of feedback bits, and it does not preserve the structure of the optimal PA vector. In the latter method, a codebook of PA vectors is shared between the source/relay and the destination, and the destination sends back the index of the best vector to the source/relay. Similar approach has been well-studied for precoding, space time codes and power loading in the context of MIMO and OFDM systems [6]-[9]. However, only few works exist on exploiting this approach on relay networks. Authors in [10] has investigated the problem of beamforming codebook design for a single MIMO-AF relay system. They showed that similar to the MIMO system case when the channel is a Rayleigh fading channel, the Grassmannian codebooks is appropriate for quantizing the optimal source beamforming vector. In contrast, Ref. [11] showed that due to the different system configuration and noise amplification at the relays, the Grasmannian codebook is no longer optimum for a system with multiple AF relays.

To the best of our knowledge, using limited feedback in a relay OFDM system has not been explored yet. One obstacle to do so has been finding a good PA solution with reasonable complexity. With a joint power constraint, we first present the optimal solution; then assuming high received SNR on either link in the relay path, we derived a sub-optimal PA solution which leads to the full capacity at high SNR but renders much 


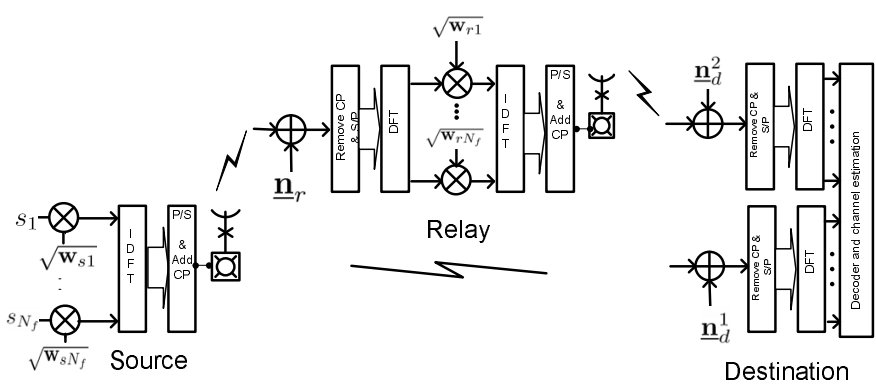

Fig. 1. A relay OFDM network with a source, a single AF relays and a destination

less complexity than other solutions in the previous works. Surprisingly, we find that when there is high received SNR in either the relay path or the direct path, the optimal solutions for both criteria reduce to simple forms. For maximizing capacity, the available power should be equally allocated to each OFDM subcarrier shared by the source and relay, while for minimum error rate, the available power should be allocated such that the received SNRs for all subcarriers at the destination are the same. We then investigate the codebook design for both the maximum capacity and minimum error rate criteria, and adapt Lloyd's algorithm to both criteria to construct the optimal codebook for use with limited feedback. Simulations show that, based on the proposed codebook construction, a good performance with mild loss can be achieved with only a few bits of feedback, and the loss becomes negligible when SNR is high.

The remainder of this paper is organized as follows: Section II describes the single OFDM-relay system. Section III presents the near-optimal solutions for PA design based on the capacity maximization criterion. Similarly in Section IV, a PA solution is provided to minimize the error rate. The numerical results and conclusion remarks are given in Section V and Section VI, respectively.

Notation: A matrix is denoted by a bold upper case letter while a vector is represented by a lower case letter. A circularly symmetric complex Gaussian random variable (RV) with mean $\mu$ and variance $\sigma^{2}$ is denoted by $z \sim \mathcal{C N}\left(\mu, \sigma^{2}\right)$.

\section{SySTEM MOdeL}

In this paper, we consider a dual-hop relay network with a pair of source/destination and a single amplify-and-forward (AF) relay using OFDM modulation. A block diagram of this system is depicted in Fig 2. We assume that OFDM with $N_{f}$ subcarriers is used in the system. Transmission takes place in two stages (two time slots). In the first stage the source sends its signal through $N_{f}$ subcarriers to the relay and destination at the same time. In the second stage, the relay retransmits an amplified version of the received signal on the same subcarrier as it has received. A fundamental question in this transmission scheme is what the amplification factor would be at each subcarrier provided that a limited power budget is available.

To formulate our problem, let the channel gains experienced by the $k$ th subcarrier between source and destination, source and relay, and relay and destination be denoted by $h_{0 k}, h_{1 k}$ and $h_{2 k}$, respectively. Given perfect pulse shaping, equalizing and sampling, at the first time slot, the received signals at the relay and at the destination for the $k$ th subcarrier are respectively represented by

$$
\begin{aligned}
& y_{r k}=h_{1 k} x_{k}+n_{r k}, \\
& y_{d k}^{(1)}=h_{0 k} x_{k}+n_{d k}^{(1)},
\end{aligned}
$$

where $n_{r k} \sim \mathcal{C N}\left(0, \sigma_{r}\right)$ and $n_{d k}^{(1)} \sim \mathcal{C N}\left(0, \sigma_{d}\right)$ are the additive noises at the relay and the destination, respectively. Given the total power constraint, we have $x_{k}=\sqrt{P_{s k}} s_{k}$ where $P_{s k}=w_{s k} P_{t o t}$ is the $w_{s k}$ portion of total power $P_{t o t}$. $\mathbf{w}_{s}=\left[w_{s 1}, \cdots, w_{s N_{f}}\right]$ is so called transmit $P A$ vector that has to be designed to optimize some performance criteria. The signals $s_{i} \mathrm{~s}, i=1, \cdots, N_{f}$, sent by the source have the unit average power.

At the second time slot, the relay retransmits its received signal, $y_{r k}$, to the destination with power $P_{r k}=P_{t o t} w_{r k}$. As a result, the received signal at the destination is

$$
y_{d k}^{(2)}=h_{2 k} \sqrt{\frac{P_{r k}}{P_{s k}\left|h_{1 k}\right|^{2}+\sigma_{r}^{2}}} y_{r k}+n_{d k}^{(2)}
$$

where $n_{d k}^{(2)} \sim \mathcal{C N}\left(\mu, \sigma_{d}^{2}\right)$ is the additive noise at the destination in the second time slot. Note that the factor $1 / \sqrt{P_{s k}\left|h_{1 k}\right|^{2}+\sigma_{r}^{2}}$ is used to normalize the received signal at the relay so that the average relay-transmit-power at the $k$ th subcarrier equals $P_{r k}$. Similar to the transmit PA vector, $\mathbf{w}_{r}=\left(w_{r 1}, \cdots, w_{r N_{f}}\right)$ is defined as the relay PA vector that must be carefully designed such that the design criterion is optimized. In this work, the criterion is either maximizing instantaneous throughput or minimizing error rate.

Through maximum ratio combining at the receiver, the total received SNR is given by summing the two consecutive received SNR, and the received SNR at the $k$ th subcarrier is given by

$$
\rho_{k}=\frac{w_{s k} a_{k} w_{r k} b_{k}}{1+w_{s k} a_{k}+w_{r k} b_{k}}+w_{s k} c_{k},
$$

where $a_{k}=\frac{P_{t o t}\left|h_{1 k}\right|^{2}}{\sigma_{r}^{2}}, b_{k}=\frac{P_{t o t}\left|h_{2 k}\right|^{2}}{\sigma_{d}^{2}}$ and $c_{k}=\frac{P_{t o t}\left|h_{0 k}\right|^{2}}{\sigma_{d}^{2}}$.

\section{PA AND LIMITED FEEDBACK DESIGN WITH MAXIMIZING CAPACITY}

In this section, we find the PA vectors $\mathbf{w}_{s}$ and $\mathbf{w}_{r}$ such that the capacity is maximized for a given channel realization. Assuming CSI is known at the receiver, the capacity of an OFDM single AF relay system is given by

$$
C\left(\mathbf{w}_{s}, \mathbf{w}_{r} \mid P_{t o t}, \mathbf{h}\right)=\frac{1}{2} \sum_{k=1}^{N_{f}} \log _{2}\left(1+\rho_{k}\right),
$$

where $\mathbf{h}$ denotes the state of the subcarriers at a particular time instant. An analytical solution for maximizing the capacity was given in [12]. However, it neglects the direct communication link between the source and the destination and hence does not achieve the full-diversity order. Furthermore, it considers power constraints on the source and relays separately. Unlike [12], we consider a more general scenario by including the direct path. 
Our optimization problem can be formulated as follow

$$
\begin{gathered}
\max _{\mathbf{w}_{\mathbf{s}}, \mathbf{w}_{\mathbf{r}}} C\left(\mathbf{w}_{s}, \mathbf{w}_{r} \mid P_{\text {tot }}, \mathbf{h}\right) \\
\text { s.t } \quad \mathbf{1}^{T} \mathbf{w}_{\mathbf{s}}+\mathbf{1}^{T} \mathbf{w}_{\mathbf{r}}=1, \\
\quad \mathbf{w}_{\mathbf{s}} \succeq 0, \quad \mathbf{w}_{\mathbf{r}} \succeq 0,
\end{gathered}
$$

where the first constraint states the joint power constraint which ensures the total power budget to allocate to the source and relay is $P_{\text {tot }}$. We have further assumed that $\mathbf{w}_{\mathbf{s}}, \mathbf{w}_{\mathbf{r}} \in$ $R_{+}^{N_{f}}$. Not only is it too difficult to show whether this problem is concave, it is also hard to present the analytical solution even if concavity is proven. To resolve this problem, by assuming that the received SNR on either link of the relay path is sufficiently high, we use an approximation of $C$ whose concavity is simply proven.

$C\left(\mathbf{w}_{s}, \mathbf{w}_{r} \mid \mathbf{h}\right) \simeq \frac{1}{2} \sum_{k=1}^{N_{f}} \log _{2}\left(1+\frac{w_{s k} a_{k} b_{k} w_{r k}}{w_{s k} a_{k}+w_{r k} b_{k}}+w_{s k} c_{k}\right)$.

We denote the right hand side (RHS) of (6) by $C^{\prime}$. Calculating the Hessian matrix of $C^{\prime}$ with respect to all $w_{r k}$ and $w_{s k}$, $k=0, \cdots, N_{f}$, shows that this matrix is always negative semidefinite meaning $C^{\prime}$ has an unique optimizer. The detailed proof is omitted here due to the space limitation, however it is provided in the upcoming journal version of this paper. provided in the appendix. The optimal PA vectors $\mathbf{w}_{s}$ and $\mathbf{w}_{r}$ that maximizes $C^{\prime}$ is obtained by mean of the Lagrange method and KKT conditions [13]. Consider the Lagrangian,

$$
\begin{aligned}
J\left(\lambda, \mathbf{w}_{s}, \mathbf{w}_{r}\right) & =\sum_{k=1}^{N_{f}} \log _{2}\left(1+\frac{w_{s k} a_{k} w_{r k} b_{k}}{w_{s k} a_{k}+w_{r k} b_{k}}+w_{s k} c_{k}\right) \\
& -\lambda\left(\sum_{k=1}^{N_{f}}\left(w_{s k}+w_{r k}\right)-1\right),
\end{aligned}
$$

where $\lambda$ is the Lagrange multiplier. By taking derivatives respect to $w_{s k}$ and $w_{r k}, k=1, \cdots, N_{f}$, and considering the KKT condition for the optimal power allocation we obtain the following equation

$$
w_{s k}=\kappa \frac{b_{k}}{a_{k}} w_{r k}
$$

where $\kappa=\frac{c_{k}+\sqrt{a_{k}\left(b_{k}-c_{k}\right)+b_{k} c_{k}}}{b_{k}-c_{k}}$. Then either of the following cases may occur;

If

$$
b_{k}>c_{k},
$$

the optimal solution would be

$$
\begin{aligned}
& w_{r k}=\left[\frac{1}{\lambda}-\left(1+\frac{1}{\kappa}\right)^{2} \cdot \frac{1}{b_{k}}\right]^{+} \frac{\kappa}{1+\kappa+(1+\kappa)^{2} c_{k} / a_{k}} \\
& w_{s k}=\kappa \frac{b_{k}}{a_{k}} w_{r k} .
\end{aligned}
$$

Otherwise

$$
w_{r k}=0, \quad w_{s k}=\left[\frac{1}{\lambda}-\frac{1}{c_{k}}\right]^{+},
$$

where $[x]^{+}=\left\{\begin{array}{lll}x & \mathrm{x} \text { is real, } & 0<x<\frac{1}{N_{f}} \\ 0 & & \text { otherwise }\end{array}\right.$ and $\lambda$ must be determined such that $\sum_{k}\left(w_{s k}+w_{r k}\right)=1$. Note that if the direct path is ignored by setting $c_{k}=0$, our general solution reduces to the solution of [12]. The condition in (8) illustrates under which circumstances using the relay is beneficial to forward the source's data on subcarrier $k$. When $b_{k}$ is stronger than the direct path $c_{k}$, using the relay is beneficial and results in higher capacity. This essentially emphasizes the importance of the strength of the relay path, $b_{k}$, over other paths. As expected, in the direct transmission, the PA solution would be simply reduced to the traditional water-filling solution.

Without loss of generality we assume $\sigma_{d}=\sigma_{r}$. Assume the total received SNR $\rho_{k}$ defined in (3) is high, $\rho_{k} \gg 1$, then $C^{\prime}$ can be further approximated as

$$
C^{\prime} \simeq \frac{1}{2} \sum_{k=1}^{N_{f}} \log _{2}\left(\frac{w_{s k} a_{k} b_{k} w_{r k}}{w_{s k} a_{k}+w_{r k} b_{k}}+w_{s k} c_{k}\right)
$$

Considering the joint power constraint in (5), we can show that $w_{s k}+w_{r k}=1 / \lambda$ for all $k$, and $\lambda=N_{f}$ regardless of what the actual CSI is. Consequently, we reach to

$w_{r k}=\left[\frac{-\left|h_{1 k}\right|^{2} \Theta_{k}+\left|h_{1 k}\right|^{2}\left|h_{2 k}\right|^{2} \Theta_{k}^{1 / 2}}{N_{f}\left(\left|h_{2 k}\right|^{2}-\left|h_{1 k}\right|^{2}\right) \Theta_{k}}\right]^{+}, w_{s k}=\frac{1}{N_{f}}-w_{r k}$

where $\Theta_{k}=\left|h_{1 k}\right|^{2}\left(\left|h_{2 k}\right|^{2}-\left|h_{0 k}\right|^{2}\right)+\left|h_{2 k}\right|^{2}\left|h_{0 k}\right|^{2}$. The PA solution in (12) gives rise to an interesting and desirable property in which unlike the PA solution in [12], $w_{s k}$ and $w_{r k}$ are determined just by knowing the channel realization of subcarrier $k$ and not other subcarriers. We will observe that the approximate solution is very close to the optimal solution in terms of performance even for all SNR values. Moreover, the result $w_{s k}+w_{r k}=1 / N_{f}$ shows that in order for (6) to be maximized at high SNR region, $1 / N_{f}$ portion of the total power must be shared by the relay and source on each subcarrier.

If the source and relay have full knowledge of CSI, the unquantized $\mathbf{w}_{s}$ and $\mathbf{w}_{r}$ can be simply determined and used in the system. However, as stated earlier, the CSI may not be available at the source/relay, and thus upon calculating the optimal PA vectors at the destination, a quantized vector is fed back to both the source and relay through a limited number of feedback bits. To send back the PA vectors by using limited feedback, $\mathbf{w}_{s}$ and $\mathbf{w}_{r}$ must be presumably restricted to lie in a finite codebook $\mathcal{W}=\left\{\left(\mathbf{w}_{s}, \mathbf{w}_{r}\right)_{1}, \cdots,\left(\mathbf{w}_{s}, \mathbf{w}_{r}\right)_{N}\right\}$, where $N$ is the size of the codebook. Given a codebook $\mathcal{W}$ and assuming it is known priori to the source/relay pair and destination, the closest vector to the optimal PA vector is drawn from $\mathcal{W}$ and its index is fed back to other parties.

Codebook design for limited feedback system has been extensively studied in the context of MIMO systems [14], [15], [8]. In the context of cooperative networks, however, very few works exist [10]. We shall use an iterative Lloyd's algorithm (LA) with a modified distortion measure to design the codebook. 
In sequel for simplicity, let's set $\mathbf{w}=\left(\mathbf{w}_{s}^{T}, \mathbf{w}_{r}^{T}\right)^{T}$ and define $\mathbf{w}_{\text {opt }}$ as the solution of (5) with $C^{\prime}$ instead of $C$. To apply LA, we use the error distance function to measure the average distortion. Hence, the average distortion $D_{\text {cap }}(\mathcal{W})$ is defined as

$$
D_{\text {cap }}(\mathcal{W})=E_{\mathbf{h}}\left[C^{\prime}\left(\mathbf{w}_{\text {opt }} \mid \mathbf{h}\right)-\max _{0 \leq i \leq N} C^{\prime}\left(\mathbf{w}_{i} \mid \mathbf{h}\right)\right] .
$$

Using this distortion function, LA can be summarized as follow:

1) First randomly generate a codebook of PA vectors $\mathcal{W}_{0}=$ $\left\{\mathbf{w}_{1}^{0}, \mathbf{w}_{2}^{0}, \cdots, \mathbf{w}_{N}^{0}\right\}$ and set $i=1$

2) Divide the set of possible channel realization vectors into $N$ quantization regions with the $k$ th region defined as

$$
\begin{aligned}
\mathcal{C}_{k}= & \left\{\mathbf{h}=\left\langle\mathbf{h}_{0}, \mathbf{h}_{1}, \mathbf{h}_{2}\right\rangle \mid C^{\prime}\left(\mathbf{w}_{l}^{i-1} \mid \mathbf{h}\right) \leq C^{\prime}\left(\mathbf{w}_{k}^{i-1} \mid \mathbf{h}\right)\right. \\
& \forall l \neq k\} .
\end{aligned}
$$

3) Construct a new codebook $\mathcal{W}_{i}$ with the $k$ th PA vector $\mathbf{w}_{k}^{i}$ given by

$$
\mathbf{w}_{k}^{i}=\underset{\mathbf{w}}{\arg \max } \mathrm{E}_{\mathbf{h} \in \mathcal{C}_{k}}\left[C^{\prime}(\mathbf{w} \mid \mathbf{h})\right] .
$$

As it is shown in [15], this step can be implemented by using the following approximation

$$
\begin{aligned}
& \underset{\mathbf{w}}{\arg \max } \mathrm{E}_{h \in \mathcal{C}_{k}}\left[\sum_{k=1}^{N_{f}} \log _{2}\left(1+\rho_{k}\right)\right] \simeq \underset{\mathbf{w}=\left(\mathbf{w}_{\mathbf{r}}, \mathbf{w}_{\mathbf{s}}\right)}{\arg \max } \\
& \sum_{k=1}^{N_{f}} \log _{2}\left(1+\frac{w_{s k} w_{r k}\left[a_{k} \bar{b}_{k}\right]}{w_{s k} \bar{a}_{k}+w_{r k} \bar{b}_{k}}+w_{s k} \bar{c}_{k}\right)
\end{aligned}
$$

where $\left[a_{k} b_{k}\right]=\mathrm{E}_{h \in \mathcal{C}_{k}}\left[a_{k} b_{k}\right], \bar{a}_{k}=\mathrm{E}_{h \in \mathcal{C}_{k}}\left[a_{k}\right]$ and $\bar{b}_{k}=$ $\mathrm{E}_{h \in \mathcal{C}_{k}}\left[b_{k}\right]$ are the conditional average coefficients. In order to determine $\mathrm{w}$ in (16), by a little change we can similarly invoke equations (9) and (10) to analytically find the optimal solution. By further simplifying (16) to a similar equation as (12).

4) If $D_{\text {cap }}\left(\mathcal{W}_{i-1}\right)-D_{\text {cap }}\left(\mathcal{W}_{i}\right)>\epsilon$, set $\mathrm{i}=\mathrm{i}+1$ and go back to the second step. Otherwise, set $\mathcal{W}=\mathcal{W}_{i}$ and terminate the algorithm

Note that while the offline design of LA may seem to be computationally complex, the realtime selection process is simple. In fact, the destination just searches over all codewords (PA vectors) in the codebook and selects the one whose associated capacity is maximum with respect to others and then sends back $B=\log _{2} N$ bits representing the codeword index to the relay/source.

In a relay system, the path loss over each link may be substantially different from one to another. This creates asymmetric channel gain on each path, and a family of codebooks will be required for the PA, with each codebook corresponding to a different set of path loss (or long-term average SNR) on the direct and relay path. Since within a small range of path loss difference, the optimal or near optimal codebook will not vary significantly, each codebook can be generated according to a quantized range of path loss (or long-term average SNR) on each path. As node's location changes slowly over time, the path loss can be updated and informed to the destination nodes through signaling. The source, relay, and destination can then adaptively update the codebook.

\section{PA AND LIMITED FEEDBACK DESIGN WITH MINIMIZING ERROR RATE}

In this section, we consider the design of $\mathbf{w}_{s}$ and $\mathbf{w}_{r}$ based on minimizing the average symbol error rate. Similar to the previous case, we first find the solution of PA problem and then apply Lloyd's algorithm on it to generate the codebook.

Given the same signal constellation is used in each subcarrier and assuming independent errors occur in each subcarriers $^{1}$, the probability of vector error rate (VER) is bounded by [9]

$$
P_{V E R} \leq 1-\prod_{k=1}^{N_{f}}\left(1-N_{e} Q\left(\sqrt{\frac{1}{2} d_{m i n}^{2} \rho_{k}}\right)\right),
$$

where $N_{e}$ is the average number of nearest neighbors to a point in any signal constellation $\mathcal{S}, d_{\min }$ is the minimum distance in $\mathcal{S}$ and $Q($.$) is the Q-function. Assuming the CSI is known$ at the receiver, by using ML detection at the destination, $\rho_{k}$ here would be the received SNR in (3). Denoting

$$
\begin{aligned}
& P_{e}\left(w_{s k}, w_{r k}, \mathbf{h}_{k}\right) \triangleq \\
& N_{e} Q\left(\sqrt{\frac{1}{2} d_{\text {min }}^{2} \gamma \underbrace{\left[w_{s k}\left|h_{0 k}\right|^{2}+\frac{w_{s k} w_{r k}\left|h_{1 k}\right|^{2}\left|h_{2 k}\right|^{2}}{1 / \gamma+w_{s k}\left|h_{1 k}\right|^{2}+w_{r k}\left|h_{2 k}\right|^{2}}\right]}_{\mu_{k}}}\right),
\end{aligned}
$$

where $\gamma=P_{t o t} / \sigma_{d}^{2}$. the optimization problem is formally expressed as

$$
\begin{array}{ll}
\min _{\mathbf{w}_{\mathbf{s}}, \mathbf{w}_{\mathbf{r}}} & 1-\prod_{k=1}^{N_{f}}\left(1-P_{e}\left(w_{s k}, w_{r k}, \mathbf{h}_{k}\right)\right) \\
\text { s.t } \quad \mathbf{1}^{T} \mathbf{w}_{\mathbf{s}}+\mathbf{1}^{T} \mathbf{w}_{\mathbf{r}}=1, & \\
& \mathbf{w}_{\mathbf{s}} \succeq 0, \quad \mathbf{w}_{\mathbf{r}} \succeq 0 .
\end{array}
$$

We again invoke the Lagrangian method and use KKT conditions to find the solution of (19). By applying similar argument as in [16] into our problem, we obtain the optimum PA vectors as

$$
\begin{aligned}
w_{s i} & =\frac{\alpha_{s i}^{2}}{\sum_{i=1}^{N_{f}}\left(\alpha_{s i}^{2}+\alpha_{r i}^{2}\right)} \\
w_{r i} & =\frac{\alpha_{r i}^{2}}{\sum_{i=1}^{N_{f}}\left(\alpha_{s i}^{2}+\alpha_{r i}^{2}\right)},
\end{aligned}
$$

where

$$
\begin{aligned}
& \alpha_{s i}=\sqrt{w_{s i}} \frac{\partial P_{e}\left(w_{s i}, w_{r i}\right)}{\partial w_{s i}}\left(1-P_{e}\left(w_{s i}, w_{r i}\right)\right)^{-1} \\
& \alpha_{r i}=\sqrt{w_{r i}} \frac{\partial P_{e}\left(w_{s i}, w_{r i}\right)}{\partial w_{r i}}\left(1-P_{e}\left(w_{s i}, w_{r i}\right)\right)^{-1}
\end{aligned}
$$

\footnotetext{
${ }^{1}$ In general, channels are correlated over adjacent subcarriers. Assuming a frequency block fading channel model, where the channel is constant within $N_{B_{C}}$ subcarriers corresponding to the coherence bandwidth, the upperbound of $P_{V E R}$ would be the same as in (17) with $N_{f}$ replaced by $N_{f} / N_{B_{c}}$.
} 
for $i=1, \cdots, N_{f}$. In the high SNR regimes (i.e $\gamma \rightarrow \infty$ ), $Q\left(\sqrt{1 / 2 d_{\min }^{2} \gamma \mu_{i}}\right)$ approaches zero, thus by substituting equations (22) and (18) into (20), we have the following equation

$$
\begin{aligned}
& 1=\frac{e^{-\frac{1}{2} d_{\text {min }}^{2} \gamma \mu_{i}}}{\mu_{i}}\left(\frac{\partial \mu_{i}}{\partial w_{s i}}\right)^{2} \times( \\
& \left.\sum_{j=1}^{N_{f}} e^{-\frac{1}{2} d_{m i n}^{2} \gamma \mu_{j}}\left[\frac{w_{s j}}{\mu_{j}}\left(\frac{\partial \mu_{j}}{\partial w_{s j}}\right)^{2}+\frac{w_{r j}}{\mu_{j}}\left(\frac{\partial \mu_{j}}{\partial w_{r j}}\right)^{2}\right]\right)^{-1} .
\end{aligned}
$$

The same equation could be derived for $w_{r j}$ in (21). From (23), we conclude that

$$
\lim _{\gamma \rightarrow \infty} \mu_{i}=\lim _{\gamma \rightarrow \infty} \mu_{j}=\text { const }
$$

for all $i, j$. Proof by contradiction is employed here to show that if (24) is violated, for at least one subcarrier the righthand side of (23) goes to zero as $\gamma \rightarrow \infty$. This result basically gives rises to a nice interpretation; in order to minimize the error rate in a single-relay OFDM system, the received SNR of all subcarriers must be equal in the high SNR regime. Since finding the solution of (23) can be so tedious, we impose another condition: $w_{s i}=w_{r i}$ for $i=1, \cdots, N_{f}$. Although the PA vectors obtained by using this simplification are not necessarily the optimal solution of (23), it significantly reduces the computational complexity while still providing satisfactory performance, as shown in Section (V). Considering (24) and (19) together, we have

$$
w_{s i}=w_{r i}=\text { const } \times(\underbrace{\left|h_{0 i}\right|^{2}+\frac{\left|h_{1 i}\right|^{2}\left|h_{2 i}\right|^{2}}{\left|h_{1 i}\right|^{2}+\left|h_{2 i}\right|^{2}}}_{\mathcal{H}_{i}})^{-1},
$$

and inserting (25) into the condition of (19) results in the value of const. Therefore, it is straightforward to show that

$$
\begin{aligned}
w_{s i}=w_{r i} & =\frac{\mathcal{H}_{i}^{-1}}{2 \sum_{j=1}^{N_{f}} \mathcal{H}_{j}^{-1}}, \\
\text { and } w_{s i}+w_{r i} & =\frac{\mathcal{H}_{i}^{-1}}{\sum_{j=1}^{N_{f}} \mathcal{H}_{j}^{-1}} .
\end{aligned}
$$

Note that $\mathcal{H}_{i}$, which we term effective channel power gain, is essentially the sum of channel power gain on the direct path and the harmonic mean of channel power gain on the relay links. Hence, (27) shows that the optimal PA on each subcarrier is achieved through effective channel power inversion.

In contrast to the capacity maximization case where $w_{s i}$ and $w_{r i}$ are determined solely based on the channel status of subcarrier $i$, in the error minimization case the whole channel information is required to compute these parameters. Therefore, using the limited feedback method described below is especially appealing.

We proceed our discussion by employing iterative Lloyd's algorithm to design an efficient codebook that quantizes the PA vectors obtained from minimizing the error rate. To do so, we again need to define a distortion measure, which is not the error distortion function in this case. Instead, following the discussion in [9], the distortion in general can be expressed and bounded as

$$
\begin{aligned}
& \mathrm{E}\left[\min _{\mathbf{w} \in \mathcal{W}}\left(\min _{1 \leq i \leq N_{f}} \mathcal{H}_{i}\left(w_{s i}-w_{i}\right)\right)^{2}\right] \leq \\
& N_{f} \mathrm{E}\left[\|\mathbf{H}\|_{2}^{2}\right] \mathrm{E}\left[\min _{\mathbf{w} \in \mathcal{W}}\left(2-2 \mathbf{w}^{T} \mathbf{w}_{s}\right)\right],
\end{aligned}
$$

where $\mathbf{w}=\left[w_{1}, \cdots, w_{N_{f}}\right], \mathbf{H}=\left[\mathcal{H}_{1}, \cdots, \mathcal{H}_{N_{f}}\right]$ and $w_{s i}$ is calculated from (26). Note that $\mathcal{H}_{i}, i=0, \cdots, N_{f}$ are always non-negative values as well as $w_{s i}$ 's. Therefore, to minimize the general distortion function, equivalently the following function can be minimized;

$$
\mathcal{D}_{V E R}(\mathcal{W})=\mathrm{E}\left[\min _{\mathbf{w} \in \mathcal{W}}\left(2-2 \mathbf{w}^{T} \mathbf{w}_{s}\right)\right] .
$$

The rest of the design procedure to quantize the optimal PA vectors are similar to what we presented in the previous section except in steps 2 and 3. Using the distortion function in (29), the $k$ th region is formed as

$$
\begin{aligned}
& \mathcal{C}_{k}=\left\{\mathbf{H}=\left\langle\mathcal{H}_{1}, \cdots, \mathcal{H}_{N_{f}}\right\rangle \mid\left(\mathbf{w}_{k}^{i-1}\right)^{T} \mathbf{w}_{s} \geq\left(\mathbf{w}_{l}^{i-1}\right)^{T} \mathbf{w}_{s}\right. \\
& \forall l \neq k\}
\end{aligned}
$$

where $\mathbf{w}_{k}^{i-1}$ is the $k$ th quantized PA vector of the codebook $\mathcal{W}_{i-1}$ constructed after $(i-1)$ th times of performing this algorithm. In the next step, the $k$ th PA vector of the new codebook $\mathcal{W}_{i}$ is given by

$$
\mathbf{w}_{k}^{i}=\frac{1}{2 \sum_{j=1}^{N_{f}} \overline{\mathcal{H}}_{j}^{-1}}\left[\overline{\mathcal{H}}_{1}^{-1}, \cdots, \overline{\mathcal{H}}_{N_{f}}^{-1}\right] .
$$

where $\overline{\mathcal{H}}_{i}=\mathrm{E}_{\mathbf{H} \in \mathcal{C}_{k}}\left[\mathcal{H}_{i}\right]$ is the conditional average entry. In the next section, we present some simulation results and verify our analytical solution through them.

\section{Simulation Results}

In this section, we use simulation to demonstrate the performance of our proposed codebooks in terms of either achieving a higher throughput or obtaining a lower error rate in an OFDM single-relay system. For simplicity of illustration, no path loss has been considered in channel simulation although the equations still hold for all channel realization. The SNR in the calculation and simulation is $\gamma=P_{\text {tot }} / \sigma_{d}^{2}$; we also assume $\sigma_{d}^{2}=\sigma_{r}^{2}$ and BPSK modulation unless we state otherwise.

In the first experiment we consider maximizing capacity to design the codebook with different sizes. We then compare their performances with a randomly-generated codebook, uniform PA scheme, where $w_{s i}=w_{r i}=1 / 2 N_{f}$ for $i=1, \cdots, N_{f}$, and the PA solution presented in [12] with evenly divided power at the source and the relay. Fig. 2 plots the normalized capacity vs. SNR (defined as $\gamma$ ) for the above mentioned schemes, where the normalized capacity is defined as the maximum achievable capacity under a specific PA scheme over the capacity of the optimal PA solution in (9) and (10). From Fig. 2 we observe that a system with a LA designed codebook outperforms a random codebook or uniform power allocation (i.e no CSI). There is also a sizeable 


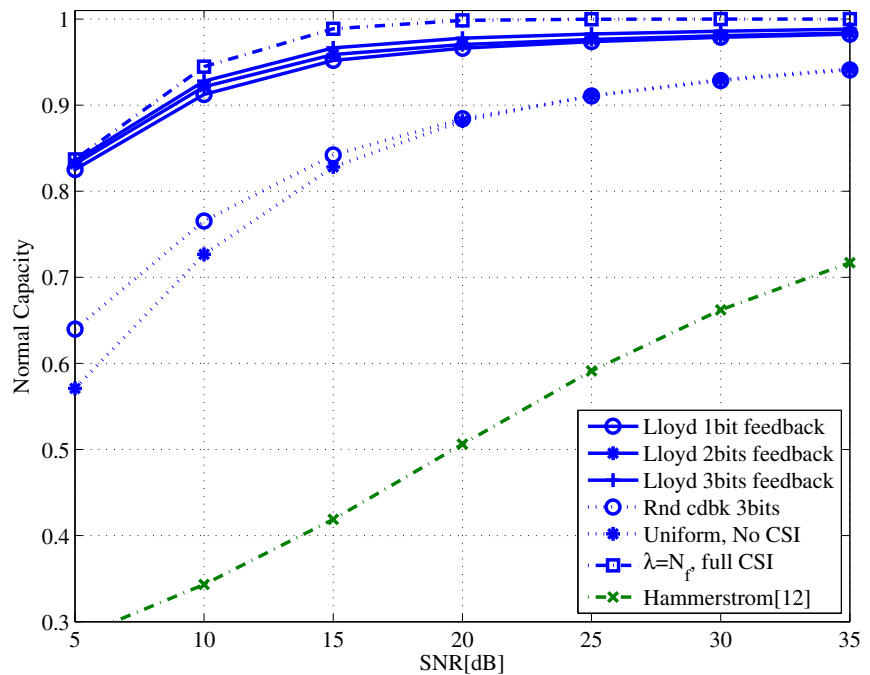

Fig. 2. Normalized capacity for a single-relay OFDM system, $N_{f}=16$, $L=3$ tabs, $\sigma_{d}=\sigma_{r}$

gap between our proposed scheme with limited feedback and the scheme presented in [12] even though full CSI availability is presumed in [12]. This difference is expected because in [12] the direct path is neglected and the power constraints on the source and destination are separate. We observe that at $\mathrm{SNR}=20 \mathrm{~dB}$, more than 95 percent of capacity can be achieved by using our design with fewer than three bits. In this figure we also realize that even with $\lambda=N_{f}$, which gives rise to a sub-optimal solution with much less complexity, we can still achieve the full capacity at high SNR. Therefore, one could use this solution to design the codebook in practice.

In Fig 3, we study the error rate performance of a codebook designed based on minimizing VER for the same system model above except with the assumption of independent subcarriers. It can be observed that the performance of 1-bit, 2-bit, and 3bit codebooks outperform the other schemes. For example, the 3-bit limited feedback PA provides a $3 \mathrm{~dB}$ improvement over uniform PA at VER $10^{-3}$. This plot also clearly demonstrates that without using a relay, the transmission diversity is reduced to 1 .

\section{CONCLUSION}

In this paper, we introduce power allocation frameworks with limited feedback in an OFDM single-relay scenario. Based on the two criteria, maximizing capacity and minimizing error rate, we derive analytical solutions for the optimal PA. We then presented the sub-optimal PA solution that is near-optimal at high SNR with significantly less computational complexity compared to previous works. Unlike other solutions, for this type of problems, our solution is a direct function of the channel realization and any convergence concerns was not the case here. Subject to the amount of feedback, we design a codebook to quantize those PA solutions for feedback. By adapting Lloyd's algorithm, we design practical codebooks for PA to provide mild to negligible performance loss from low to high SNR with very low feedback overhead.

\section{REFERENCES}

[1] Z. Sahinoglu and P. Orlik, "Regenerator versus simple-relay with optimum transmit power control for error propagation," IEEE Commun. Lett., vol. 7, no. 9, pp. 416-418, Sept. 2003.

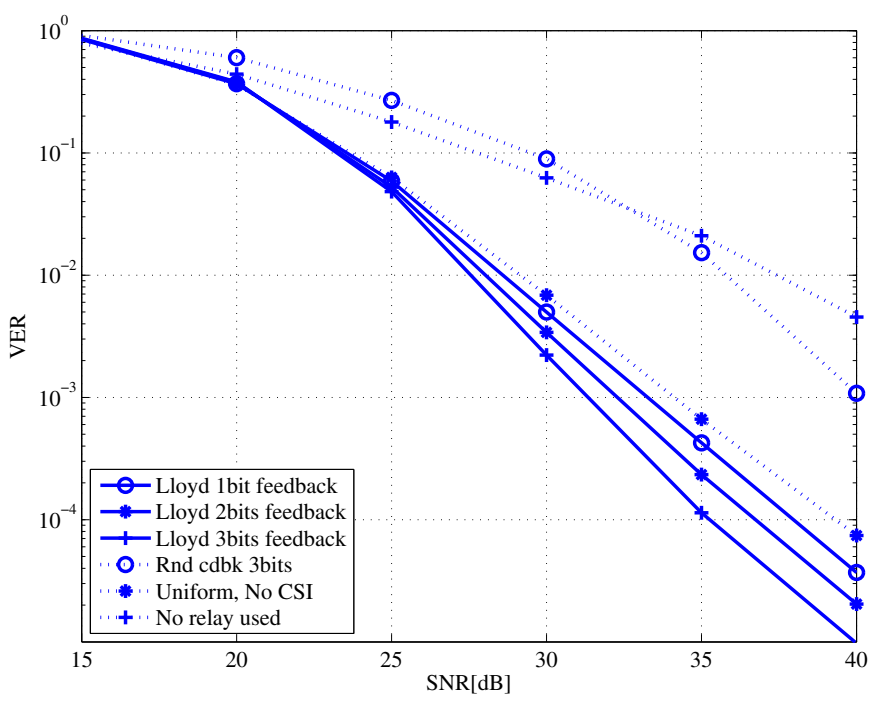

Fig. 3. Vector error rate performance for a single-relay OFDM system with limited feedback, $N_{f}=16, \sigma_{d}=\sigma_{r}$, independent subcarriers

[2] M. O. Hasna and M. S. Alouini, "Optimal power allocation for relayed transmissions over rayleigh-fading channels," IEEE Transactions on Wireless Communications, vol. 3, no. 6, pp. 1999-2004, Nov. 2004.

[3] I. Hammerstrom, M. Kuhn, and A. Wittneben, "Impact of relay gain allocation on the performance of cooperative diversity networks," in Proc. IEEE Vehicular Technology Conf. (VTC), vol. 3, Sept. 2004, pp. 1815-1819.

[4] Z. Yi and I.-M. Kim, "Joint optimization of relay-precoders and decoders with partial channel side information in cooperative networks," IEEE Journal on Selected Areas in Communications, vol. 25, no. 2, pp. 447458, Feb. 2007.

[5] N. Ahmed, K. A., and A. Sabharwal, "Outage minimization with limited feedback for the fading relay channel," IEEE Trans. Commun., vol. 54, no. 3, pp. 574-574, Mar. 2006.

[6] D. J. Love and R. W. Heath, "Grassmannian beamforming on correlated MIMO channels," in Proc. IEEE Global Telecommn. Conf. (GLOBECOM), vol. 1, Dallas, TX, USA, 2004, pp. $106-110$.

[7] J. Choi and R. W. Heath, "Interpolation based transmit beamforming for MIMO-OFDM with limited feedback," IEEE Trans. Acoust., Speech, Signal Processing, vol. 53, no. 11, pp. 4125-4135, Nov. 2005.

[8] T. Pande, D. J. Love, and J. V. Krogmeier, "Reduced feedback MIMOOFDM precoding and antenna selection," IEEE Trans. Acoust., Speech, Signal Processing, vol. 55, pp. 2284-2293, May 2007.

[9] D. J. Love and R. W. Heath, "OFDM power loading using limited feedback," IEEE Trans. Veh. Technol., vol. 54, no. 5, pp. 1773-1780, Sept. 2005.

[10] B. Khoshnevis, W. Yu, and R. Adve, "Grassmannian beamforming for MIMO amplify-and-forward relaying," in Annual Conf. on Information Sciences and Systems, Princeton, NJ, USA, Mar. 2008, pp. 161-166.

[11] Y. Zhao, R. Adve, and T. J. Lim, "Beamforming with limited feedback in amplify-and-forward cooperative networks," in Proc. IEEE Global Telecommn. Conf. (GLOBECOM), Nov. 2007, pp. 3457-3461.

[12] I. Hammerstrom and A. Wittneben, "On the optimal power allocation for nonregenerative OFDM relay links," in Proc. IEEE Int. Conf. Communications (ICC), vol. 10, June 2006, pp. 4463-4468.

[13] S. Boyd and L. Vandenberghe, Convex Optimization. Cambridge University Press, March.

[14] D. Love and R. Heath, "Limited feedback unitary precoding for orthogonal space-time block codes," IEEE Trans. Signal Processing, vol. 53, no. 1, pp. 64 - 73, Jan. 2005.

[15] V. Lau and T. Wu, "Optimal transmission and limited feedback design for OFDM/MIMO systems in frequency selective block fading channels," IEEE Trans. Wireless Commun., vol. 6, no. 5, pp. 1569-1573, May 2007.

[16] L. Goldfeld, V. Lyandres, and D. Wulich, "Minimum BER power loading for OFDM in fading channel," IEEE Trans. Commun., vol. 50, no. 11, pp. 1729-1733, Nov. 2002. 